\title{
Lexical and sublexical knowledge influences the encoding, storage, and articulation of nonwords
}

\author{
Gary Jones • Hannah L. Witherstone
}

Published online: 19 November 2010

(C) Psychonomic Society, Inc. 2010

\begin{abstract}
Nonword repetition (NWR) has been used extensively in the study of child language. Although lexical and sublexical knowledge is known to influence NWR performance, there has been little examination of the NWR processes (e.g., encoding, storage, and articulation) that may be affected by lexical and sublexical knowledge. We administered two- and three-syllable spoken nonword recognition and nonword repetition tests on two independent groups of 31 children (mean age $=5$ years 7 months). Spoken nonword recognition primarily involves encoding and storage, whereas NWR involves an additional articulation process. The influence of lexical and sublexical knowledge was determined by examining the number of lexical errors produced. There was clear involvement of long-term lexical and sublexical knowledge in both spoken nonword recognition and NWR. In spoken nonword recognition, twice as many errors involved selecting a foil that contained a lexical item (e.g., 'yashukup') as involved selecting a foil that contained only nonsense syllables (e.g., 'yashunup'). In repetition, over 30\% of errors changed a nonsense syllable to a lexical item. Our results show that long-term lexical and sublexical knowledge is pervasive in NWR. Any explanation of NWR performance must therefore consider the influence of lexical and sublexical knowledge throughout the whole repetition process, from the encoding to the articulation of nonwords.
\end{abstract}

Keywords Nonword repetition · Encoding · Articulation · Phonological working memory $\cdot$ Lexical knowledge

G. Jones $(\bowtie) \cdot$ H. L. Witherstone

Nottingham Trent University,

Nottingham, England

e-mail: gary.jones@ntu.ac.uk
If children are to attain an accomplished use of their native language, they must first learn the vocabulary of that language. Vocabulary learning is at least a two-stage process. The first stage is learning the word boundaries from incoming speech that does not ordinarily contain pauses. The second stage is the learning of words in a meaningful way. Word learning involves the temporary storage of a word in phonological working memory, where learning processes can act upon the word for subsequent storage in long-term memory and lexical access processes can retrieve the word for later articulation. The present report focuses on the second stage of vocabulary learning. Extensive research (e.g., Dollaghan, Biber, \& Campbell, 1995; Gathercole \& Adams, 1993; Munson, Kurtz, \& Windsor, 2005a) has been conducted on word learning using nonword repetition (NWR), where nonwords varying along a number of dimensions (e.g., stress, length) are presented to the child, who must repeat the nonwords aloud. The task is an extremely good predictor of language ability (e.g., Cheung, 1996; Gathercole \& Baddeley, 1989) and involves the ability to encode, store, and articulate the spoken nonword, amongst other processes (Coady \& Aslin, 2004).

\section{Nonword repetition research}

NWR tests were originally designed to examine phonological working memory, a temporary store for auditory information. Research showed that adults with impaired phonological working memory had difficulty learning wordnonword pairs relative to word-word pairs (Baddeley, Papagno, \& Vallar, 1988). Words would be expected to have some form of representation in long-term memory, whereas nonwords would not, leading to the belief (at the time) that 
impaired performance on word-nonword pairs was primarily due to nonword learning being reliant on phonological working memory. Gathercole and Baddeley (1989) supported the phonological working memory account by finding that 4- to 5-year-olds' NWR performance declined as nonword length increased. Longer nonwords place more of a burden on phonological working memory and therefore have an increased probability of being incorrectly repeated. Subsequent research showed further support by finding nonword length effects for 2- to 3-year-olds (Gathercole \& Adams, 1993) and 7- to 11-year-olds (Archibald \& Gathercole, 2006).

However, it quickly became clear that even phonologically novel words had strong links to long-term lexical and sublexical knowledge. For the purposes of this article, lexical knowledge refers to phoneme sequences that correspond to a known lexical item or morpheme, and sublexical knowledge refers to phoneme sequences that form part of a known lexical item or morpheme. Gathercole (1995) reanalysed children's responses to her set of nonwords on the basis of adult ratings of the wordlikeness of each nonword. Children showed superior repetition accuracy for nonwords that adults rated high in wordlikeness over nonwords that adults rated low in wordlikeness. The nonwords that adults rated high in wordlikeness showed a greater number of lexical items or morphemes (e.g., 'thickery', 'sladding') than did the nonwords that adults rated low in wordlikeness (e.g., 'glistow', 'woogalamic'). Wordlikeness effects have also been shown for other sets of nonwords (e.g., Frisch, Large, \& Pisoni, 2000; Munson et al., 2005a).

There is also a sublexical influence on repetition performance. Nonwords constructed from high-frequency phoneme sequences are easier to repeat than nonwords constructed from low-frequency phoneme sequences (e.g., Munson, 2001; Vitevitch, Luce, Charles-Luce, \& Kemmerer, 1997). Although frequency and wordlikeness have been shown to have a strong relationship (Frisch et al., 2000), they have also been shown to exert independent influences on repetition performance (Munson, Edwards, \& Beckman, 2005a).

In order to test the influence of phonological working memory, any help from long-term lexical and sublexical knowledge needs to be minimised. Munson et al. (2005a) therefore suggested that nonwords be constructed from lowfrequency phoneme sequences that do not contain any lexical items or morphemes. However, since low-frequency phoneme sequences clearly exist in spoken language, their components are not precluded from having some form of representation as long-term sublexical knowledge. Frisch et al. (2000) also suggested that even nonwords comprising low-frequency phoneme sequences can still activate lexical knowledge.
Gathercole and colleagues (e.g., Gathercole, 1995, 2006; Gathercole, Frankish, Pickering, \& Peaker, 1999) used redintegration (Schweickert, 1993) as an explanation for the influence that lexical and sublexical knowledge has on NWR. Any spoken input is placed in phonological working memory, where it decays after approximately $2,000 \mathrm{~ms}$ unless it is rehearsed (Baddeley \& Hitch, 1974). If the information in phonological working memory becomes degraded in quality (e.g., if a large amount of information is placed in phonological working memory or the information remains there for longer than $2,000 \mathrm{~ms}$ ), two processes can be applied to help repair the degraded information. First, lexical knowledge can be used to 'fill in' the missing parts. For example, if only the initial phoneme of the nonword 'trumpetine' was degraded, knowledge of the lexical item 'trumpet' could repair the / $t$. Second, sublexical knowledge, in the form of phonological similarity, can be used to repair degraded information. ${ }^{1}$ For example, the final phoneme $/ \mathrm{k}$ / of the nonword 'neek' could be repaired because the nonword rhymes with 'week'. Note that when a spoken input becomes degraded in quality, it can still retain some information. For example, if the $/ b /$ of the nonword 'rubid' became degraded in quality but retained the fact that it was a consonantal stop, it could be correctly repaired using the lexical item 'bid' or incorrectly repaired using the lexical items 'did' and 'kid'. However, it could not be repaired using lexical items such as 'lid' and 'hid', since they do not begin with a consonantal stop.

Redintegration was originally used to explain the greater serial recall accuracy of word lists over nonword lists (Hulme, Maughan, \& Brown, 1991). The application of redintegration to NWR (using the original conception of redintegration described above) is appropriate because nonwords involve a sequence of sounds that are initially unknown to the listener, and hence nonword processing can be viewed as serial recall of a phoneme sequence. When viewed as such, NWR shows the same type of primacy and recency effects that are encountered in the serial recall of words (Gupta, 2005), and overall NWR performance shows a strong relationship to serial recall performance (Gupta, 2003).

The redintegration account makes specific predictions regarding NWR performance: (1) Redintegration is most likely to benefit nonwords that are wordlike or that contain high-frequency phoneme sequences, because they have more in common with existing lexical and sublexical knowledge, and (2) as the quality of a nonword held in phonological working memory decreases, the likelihood of

\footnotetext{
${ }^{1}$ Although Schweickert (1993) does not explicitly state that sublexical information is used to repair degraded information, he does indicate its use: "detailed knowledge of the features of phonemes in the context of other phonemes would be useful" (p. 173).
} 
long-term lexical and sublexical involvement increases. Prediction 1 has already been supported by the wordlikeness and frequency research reported above. It is also supported in a broader capacity, however, by Dollaghan et al. (1995), who found improved NWR performance for nonwords containing lexical items as opposed to nonwords without (e.g., 'bathesis' vs. 'fathesis'), consistent with the idea that lexical knowledge is more beneficial as nonwords become more wordlike. However, Dollaghan et al. also found that errors in NWR often involved substituting a nonsense syllable for a lexical syllable, consistent with the idea that lexical (and possibly sublexical) knowledge is used to repair the decayed representation of nonwords. To our knowledge, Prediction 2 has not been directly tested and is one focus of the present study. The quality of a stored nonword in phonological working memory can be manipulated by varying the length of the nonword, since long nonwords are more prone to decay than short nonwords. Taking into account the results of Dollaghan et al., as nonword length increases, one should therefore also expect an increase in NWR errors that replace a nonsense syllable with a lexical item, due to the increased involvement of lexical (and possibly sublexical) knowledge.

The clear indication from the literature covered thus far is that NWR performance is influenced by both phonological working memory and long-term lexical and sublexical knowledge. However, there are three key processes involved in NWR (other processes may be involved, but we consider the following the key processes, as do Coady \& Aslin, 2004): accurate perception and encoding of the spoken nonword, with its temporal sequence; efficient storage of the phoneme sequence; and preparation and execution of speech-motor processes to articulate the phoneme sequence. For the purposes of the present study, we combine the first two processes, such that NWR involves encoding/storage and articulation. Since tests of NWR performance examine the child's articulated response (i.e., after encoding/storage and articulation have taken place), it is unclear whether the interaction between phonological working memory and long-term lexical and sublexical knowledge occurs at the encoding/storage stage, the articulation stage, or both. The redintegration account predicts no lexical or sublexical involvement at the stage of encoding/storage, since redintegration occurs at the later point of retrieval (e.g., Gathercole, 1995; Thorn, Gathercole, \& Frankish, 2005; see also Hulme et al., 1997). That is, redintegration is hypothesised to occur at a late stage in NWR - when the nonword is retrieved from memory for articulation - rather than at an early stage, such as during encoding of the incoming sound patterns.

If a nonword task is administered that does not involve articulation, the influence that long-term lexical and sublexical knowledge has on encoding/storage can be examined. The results of the encoding/storage nonword task can then be compared with the influence that long-term lexical and sublexical knowledge has on the traditional nonword repetition task, which involves encoding/storage and articulation. While we accept that the 'additive' nature of this hypothesis suggests that encoding, storage, and articulation are sequential processes, any differences in performance between the articulation-excluded and articulation-included nonword tasks nevertheless must indicate additional processes that are involved in articulation over and above the ability to encode and store a nonword.

Encoding/storage and articulation have been compared previously within the context of word production. For example, Rossmeissl and Theios (1982) recorded response times in word production in which participants read aloud words either immediately or after a delay. It was assumed that during a delay, participants can encode and store the material, and therefore the delay condition should only involve articulation. There was a large reduction in response latencies in the delay condition, suggesting that the no-delay condition involved encoding, storage, and articulation, whereas only articulation was involved in the delay condition. Balota and Chumbley (1985) further examined the effect of a delay until word production. By manipulating word frequency, they found that highfrequency words were produced more quickly than lowfrequency words under various temporal delays. Taken together, these two studies illustrate that the articulation process must involve long-term lexical (and possibly sublexical) knowledge, since frequency effects emerged when both encoding and storage were minimised. However, studies of spoken word recognition indicate that long-term lexical (and possibly sublexical) knowledge is also involved in encoding (Norris, McQueen, \& Cutler, 2003). Recognising a word primarily involves lexical access and encoding, with minimal need for storage and no requirement for articulation. Word recognition studies show superior response times for high-frequency words over low-frequency words (e.g., Brysbaert, Lange, \& Wijnendaele, 2000; Forster \& Chambers, 1973). For word stimuli at least, long-term lexical (and possibly sublexical) knowledge must influence both encoding and articulation.

However, encoding/storage and articulation have not been examined thoroughly in the production of nonwords, yet without such an examination we cannot be certain where interactions between phonological working memory and long-term lexical and sublexical knowledge may lie. We suggest that two tasks are required to examine the influence of lexical and sublexical knowledge on both encoding/storage and articulation. Conducting a spoken nonword recognition task on an item-by-item basis 
excludes articulation, emphasising the role of encoding/ storage (other processes will also be involved, but encoding/storage should be primary). Conducting a nonword repetition task includes articulation, emphasising any additional processing over and above encoding/storage.

\section{Spoken nonword recognition research}

Although the designs of some studies have included several to-be-remembered spoken nonwords presented for later recognition (e.g., Gathercole \& Pickering, 1999), we know of no study that has examined spoken nonword recognition on an item-by-item basis in a way that is analogous to NWR. One possible exception is Swingley and Aslin (2007), who trained 1.5-year-olds on spoken nonwords that related to specific pictures before a later item-by-item recognition test (conducted by examining gaze duration when presented with the associated picture and an unassociated one, together with the spoken nonword). For our purposes, the most pertinent spoken nonword recognition study is that of Frisch et al. (2000), who examined serial spoken nonword recognition in adults. Participants rated 72 nonwords for wordlikeness, before being given a recognition test involving the original 72 nonwords plus 72 foils. Providing support for long-term lexical and sublexical influences in encoding/storage, Frisch et al. found improved spoken nonword recognition performance for nonwords that contained high-frequency phoneme sequences relative to nonwords that contained low-frequency phoneme sequences. Interestingly, no effect of nonword length on spoken nonword recognition was found-consistent with research in spoken word recognition (e.g., Frederikson \& Kroll, 1976), but in contrast to the NWR literature, in which researchers consistently find length effects (e.g., Gathercole \& Baddeley, 1989; Jones, Gobet, \& Pine, 2007; Jones, Tamburelli, Watson, Gobet, \& Pine, in press).

The literature on speech perception also supports longterm lexical and sublexical influences, at least for the encoding of incoming sounds. By examining ambiguous sounds in Dutch, Norris et al. (2003) found that the categorisation of the ambiguous sound was dependent upon the lexical context of the stimuli. For example, the sound of the final /f/ in druif (grape) was altered to occur between /f/ and /s/ on a continuum but was almost always perceived as /f/ because of its context in relation to the remainder of the word. Pitt and McQueen (1998) showed that sublexical knowledge also affected the processing of English-based nonwords. By manipulating the transitional probabilities of vowel-consonant biphones, they found that ambiguous consonants were more likely to be perceived as the consonant that most often followed the vowel in English.

\section{The present study}

We conducted spoken nonword recognition and NWR on independent groups of 5- to 6-year-olds, using the same set of two- and three-syllable nonwords for each task. The two tasks were designed to assess the long-term lexical and sublexical involvement in encoding/storage and articulation and to test the redintegration predictions outlined earlier. We used 5- and 6-year-old children for two reasons. First, a pilot study showed that 5- to 6-year-old children are old enough to successfully complete both spoken nonword recognition and nonword repetition, yet at the same time we know that children of this age are unable to reliably use any memory strategies such as rehearsal (Gathercole \& Adams, 1994). This is important because both tasks, in part, required the child to remember nonwords. Minimal use of memory strategies would therefore result in more reliance on phonological working memory and lexical/sublexical knowledge when completing the tasks. Second, at 5-6 years of age, both phonological working memory and lexical/ sublexical knowledge are strong predictors of vocabulary learning. Up to the age of 5, the best predictor of vocabulary learning is phonological working memory (Gathercole \& Baddeley, 1989), and at age 6 and above, the best predictor is lexical/sublexical knowledge (Gathercole, Willis, Emslie, \& Baddeley, 1992).

The spoken nonword recognition task involves presenting each nonword followed by three further nonwords - the same (target) nonword, a lexical foil that substitutes one phoneme of the target nonword to create a syllable that is a lexical item, and a nonlexical foil that substitutes one phoneme of the target nonword to create another nonsense syllable. The NWR task then involves presentation of the original nonwords for the children to repeat aloud. A forced pause is introduced between hearing the nonword and repeating it that is equivalent to the time lapsed between hearing the original nonword and hearing the target in the spoken nonword recognition test.

For spoken nonword recognition, the use of lexical foils allowed us to examine long-term lexical and sublexical involvement in encoding/storage. If there is no such longterm involvement, any errors on the task should involve equal numbers of lexical and nonlexical foil selections. For NWR, repetition errors that change a nonsense syllable into a lexical item show the clearest influence of lexical and sublexical knowledge. We use the term lexical error to denote the selection of a lexical foil in the spoken nonword recognition task and the replacement of a nonsense syllable with a lexical item in the NWR task. An increase in lexical errors in the repetition task over the spoken nonword recognition task therefore indicates the likely involvement of long-term lexical and sublexical knowledge in the articulation process. 
The design of the present study allowed us to examine several research questions that have not yet been examined in the domain of nonword processing:

1. The extent to which encoding/storage and articulation are influenced by lexical and sublexical knowledge. Current NWR studies indicate that lexical and sublexical knowledge influences repetition performance, but as yet we do not know whether this occurs primarily at the encoding/storage stage, the articulation stage, or both. We can answer this question by comparing the number of lexical errors produced in spoken nonword recognition with the number of lexical errors produced in NWR.

2. Whether the influence of lexical and sublexical knowledge increases as the quality of a stored nonword decreases. The redintegration account predicts that for NWR, lexical and sublexical knowledge exerts a greater influence as the quality of a nonword declines. As the influence of lexical and sublexical knowledge increases, so should the proportion of repetition errors that are classified as lexical errors. We can answer this question by (for example) comparing the proportion of errors that are classed as lexical errors across two- and threesyllable nonwords, the latter of which are more prone to decay due to their phonemic length. If a similar pattern of results also occurs for spoken nonword recognition, then redintegration, or another similar process, must occur at an earlier stage than retrieval.

3. The extent to which errors in NWR are explained by encoding/storage, and the extent to which they are explained by articulation. Currently, studies of NWR have based their results on repetition accuracy, which we know involves encoding/storage and articulation. Hence, there is no way of determining whether the source of NWR errors lies with encoding/storage or with articulation. We can answer this question by comparing accuracy in spoken nonword recognition with accuracy in NWR, and thus begin to identify the relative contributions of encoding/storage and articulation in NWR performance.

\section{Method}

\section{Participants}

A total of 64 children were recruited from three primary schools within a 9-mile radius north of Nottinghamshire. Two children were removed from the analyses as they failed to perform within normal ranges on the British Picture Vocabulary Scale-Version 2 (BPVS-II; Dunn, Dunn, Whetton, \& Burley, 1997). The remaining 62 children ( 28 male, 34 female; age range $=5$ years 3 months to 6 years 9 months, $M=5$ years 7 months) were native speakers of English and had no learning, speech, or hearing difficulties, as reported by their school teacher.

\section{Materials}

Creation of nonwords Fifteen two-syllable (5-7 phonemes in length) and 15 three-syllable (7-8 phonemes in length) nonwords were created. In order for all nonwords to be perceived as plausible English words, we ensured that (1) the nonwords followed the predominant stress pattern for two-syllable words (strong-weak; e.g., geris ['ger Is]), and one of the predominant stress patterns for three-syllable nonwords (strong-weak-weak; e.g., heburin ['hebərIn]); and (2) all of the constituent biphones within each nonword existed in the child's vocabulary. We extracted biphone frequencies from the Children's Printed Word Database (CPWD, a database containing frequency counts of the printed word vocabulary of 5- to 9-year-old children; Masterson, Stuart, Dixon, \& Lovejoy, 2010) and ensured that each biphone had a frequency of at least 10. Although the CPWD is a summary of children's written word frequencies, as far as we are aware no such database exists for children's spoken English. Note that we ensured that all nonwords were nonlexical by constructing nonwords that contained no grammatical morphemes and no syllables that existed as lexical items in the CPWD.

In order for the spoken nonword recognition test to be of sufficient difficulty, we used only nonwords that contained low-frequency biphones. We compared the constituent biphones of the nonwords with the biphones from a random sample of 30 of the lowest-frequency ( 3 occurrences per million) words in the CPWD (matched for stress pattern, length in syllables, and length in phonemes). For twosyllable items, the nonword biphones had a significantly lower average $\log$ frequency $[M=.43$ vs. $.54 ; t(28)=7.13$, $p<.001]$. The same was true of three-syllable nonwords $[M=.47$ vs. $.57 ; t(28)=5.59, p<.001]$. (Average log frequency was computed using the method of Luce and colleagues: Jusczyk, Luce, \& Charles-Luce, 1994; Vitevitch et al., 1997; Vitevitch \& Luce, 1998.) Note that by using nonwords containing low-frequency phoneme sequences and having no lexical or morphological components, we minimised the influence of lexical and sublexical knowledge. If lexical errors still persisted in our spoken nonword recognition and NWR results, the influence of lexical and sublexical knowledge then would likely be prevalent for all types of nonword.

Creation of foils The spoken nonword recognition test had each nonword succeeded by three further nonwords: the original nonword (the target), a lexical foil, and a 
nonlexical foil. The lexical and nonlexical foils for each nonword were created by changing the initial phoneme of the last syllable to create either a final syllable that was a lexical item in the CPWD or a final syllable that was a nonsense syllable different from that in the target. For example, the nonword 'yashudup' had the lexical foil 'yashukup' and the nonlexical foil 'yashunup'. The phoneme change could differ by place of articulation, manner of articulation, voice, or a combination of these. In the example, /d/ and /k/ differ by place and voice, and /d/ and $/ \mathrm{n} /$ differ only by manner of articulation; since here the phoneme used to create the lexical syllable differs by two features from the original phoneme, whereas the phoneme used to create the nonlexical syllable differs by one feature, the lexical syllable might be easier to distinguish from the original. We therefore ensured that foil selections were not influenced by the number of phoneme features that changed when creating nonlexical and lexical syllables [nonlexical phoneme features, $M=2.13, S D=.75$; lexical phoneme features, $M=2.16, S D=.72 ; t(28)=0.17, p=.869]$.

Position of the stimuli For the spoken nonword recognition test, the 15 nonwords at each syllable length were randomly split into three groups of 5 nonwords. The first group had the target in the first position, the second group had the target in the second position, and the third group had the target in the third position. The relative positions of the lexical and nonlexical foils were counterbalanced (e.g., when the target was in the first position, the lexical foil occurred in Position 2 twice and in Position 3 thrice).

There was no difference between the nonwords at each position in terms of number of phonemes, spoken duration, or average $\log$ frequency ( $p=.25$ or higher). We also ensured that the nonwords at each length and position contained similar numbers of neighbours. All neighbours were totalled for each syllable in a nonword (neighbours being defined as words within the CPWD that differed by only one phoneme from the nonsense syllable). There was no difference in the number of neighbours for nonwords in each position for both two-syllable nonwords $[F(2,12)=$ $0.03, M S E=71.57, p=.972]$ and three-syllable nonwords $[F(2,12)=0.29, M S E=39.07, p=.765]$.

Recording of the stimuli The nonwords and their foils were recorded onto a Sony ICD-MX20 digital voice dictaphone by the second author, who is native to Nottingham. All nonwords were converted to MP3 format using Sony Digital Voice Editor, Version 3.1 (available from http:// esupport.sony.com/). The nonwords were edited using Audacity (www.audacity.sourceforge.net), such that the original nonword was followed by a pause of $1.2 \mathrm{~s}$, followed by the foils and target separated by 0.7 -s pauses. The two-syllable nonwords had a mean spoken duration of
$0.8 \mathrm{~s}$, and therefore the temporal durations until hearing the target nonword were 2.0, 3.5, and $5.0 \mathrm{~s}$ when the target appeared in the first, second, and third positions, respectively $(2.2,3.9$, and $5.6 \mathrm{~s}$ for the three-syllable nonwords, which had a mean spoken duration of $1.0 \mathrm{~s}$ ). The order of the sets of nonwords plus foils was randomised to produce two sound files for each nonword length: the randomised order and the reverse of this order.

For the NWR test, each nonword was succeeded by a pause and then a beep (the children were asked to wait for the beep before repeating the nonword). The pause duration matched the temporal duration until the target of the relevant nonword was heard in the spoken nonword recognition test. For example, the target for the nonword 'virel' appeared in the first position, and therefore the nonword 'virel' was followed by a 1.7 -s pause and a 0.3 -s beep in the NWR test. For each nonword length, two sound files were produced that matched the randomised order of nonwords in the spoken nonword recognition test. Figure 1 gives an illustration of the presentation of stimuli for both spoken nonword recognition and NWR.

\section{Design}

The type of test (spoken nonword recognition, NWR) was a between-subjects variable, and nonword length (two syllables, three syllables) was a within-subjects variable. For spoken nonword recognition, the position of the target (1st, 2nd, 3rd) was also a within-subjects variable; for NWR, the pause duration $(2.0,3.5$, and $5.0 \mathrm{~s}$ for two-syllable stimuli; $2.2,3.9$, and $5.6 \mathrm{~s}$ for three-syllable stimuli) was also a within-subjects variable.

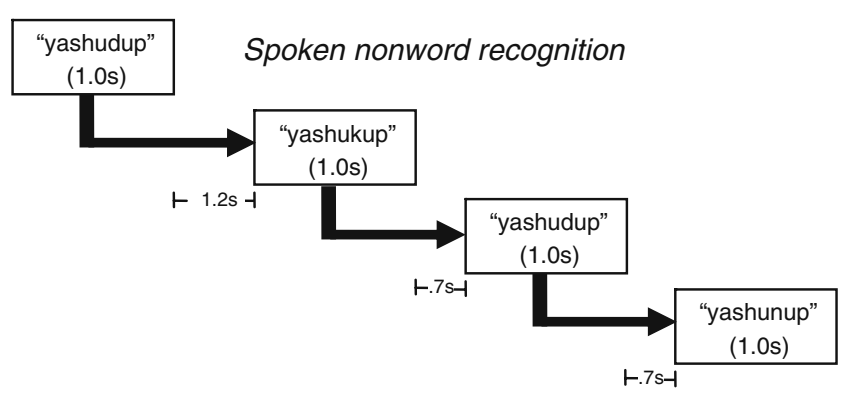

Nonword repetition

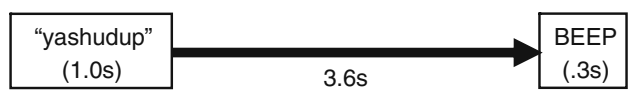

Fig. 1 Example of the presentation of stimuli for both spoken nonword recognition and nonword repetition. In the example, the target foil appears as the second of the three foils, so the pause length for nonword repetition is adjusted to cater for this 


\section{Procedure}

All children were tested individually in a quiet area of their school. The sound files were played to each child using a Sony ICD-MX20 digital voice dictaphone connected to Creative Inspire T10 portable speakers.

Children were randomly allocated to either the spoken nonword recognition group or the NWR group and given standardised instructions together with an example trial (the instructions and example differed depending on whether spoken nonword recognition or NWR was being carried out). Once the child had completed the example and had confirmed to the experimenter that they understood the task, the testing began.

The session for each child consisted of all stimuli at one nonword length, followed by the BPVS-II, followed by all stimuli at the other nonword length. The presentation order of the two-syllable and three-syllable stimuli was counterbalanced.

For spoken nonword recognition, the child was required to say whether the original nonword matched the first nonword that they heard, the second, or the last. Children's responses were noted by the experimenter. For NWR, the child repeated the nonword as accurately as possible after hearing the beep. Responses were recorded onto a Sony ICD-MX20 digital voice dictaphone for later transcription.

\section{Results and discussion}

Below we first analyse the results for spoken nonword recognition, followed by the nonword repetition results, and finally a comparison between the two. Note that the children in the spoken nonword recognition condition and the children in the NWR condition had very similar levels of vocabulary knowledge, as evidenced by their standardised BPVS-II scores [spoken nonword recognition, $M=$ 101.87, $S D=10.95$; nonword repetition, $M=103.13, S D=$ $11.20 ; t(60)=0.45, p=.656]$.

Spoken nonword recognition

During the collection of the data, a problem was discovered with one of the nonwords, 'reldufop', the nonword foil for which was 'relduthop'. The recording of the nonword foil resulted in the phoneme /th/ being almost indistinguishable from /f/, and therefore 'reldufop' was removed from all analyses.

Verification of data Participants' selection of the target was significantly above chance levels at each position of the target and for each nonword length (all $p \mathrm{~s}<.001$ ), indicating that the children were not simply guessing among the three options.

Analyses Figure 2 shows the percentage selection of the target (i.e., the original nonword), lexical foil, and nonlexical foil at each position and for each nonword length. A 2 (nonword length: two or three syllables) $\times 3$ (position: 1, 2, or 3) $\times 3$ (foil type: target, lexical, nonlexical) repeated measures ANOVA was carried out on the data. Note that by including foil type as a variable, no independent effects of nonword length, position, and the interaction between the two are seen (collapsing the data across foil types will always total $100 \%$, since it includes the percentage selections of the target and the lexical and nonlexical foils). There was a significant effect of foil type $\left[F(2,60)=114.44, p<.001, \eta_{\mathrm{p}}{ }^{2}=.79\right]$, with post-hoc Bonferroni tests indicating that the target was selected more often than both the lexical and nonlexical foils (both $p \mathrm{~s}<$ .001), and lexical foils were selected more often than nonlexical foils $(p<.001)$. There was also a significant interaction between nonword length and foil type $[F(2,60)=$ $\left.10.17, p<.001, \eta_{\mathrm{p}}{ }^{2}=.25\right]$. We confirmed that the interaction reflected not only a higher number of target selections for two-syllable stimuli over three-syllable stimuli $[t(30)=3.46$, $p=.002]$, but also a lower number of lexical foil selections for two-syllable stimuli over three-syllable stimuli.

The interaction between position and foil type only approached significance $\left[F(4,120)=2.38, p=.056, \eta_{\mathrm{p}}{ }^{2}=\right.$ $.07]$, indicating a slight decrease in target selections (and hence an increase in foil selections) at Position 2. The position of the target did not significantly influence its recognition. The three-way interaction was not significant $\left[F(4,120)=0.39, p=.815, \eta_{\mathrm{p}}{ }^{2}=.01\right]$.

The spoken nonword recognition results confirm the involvement of phonological working memory and long-

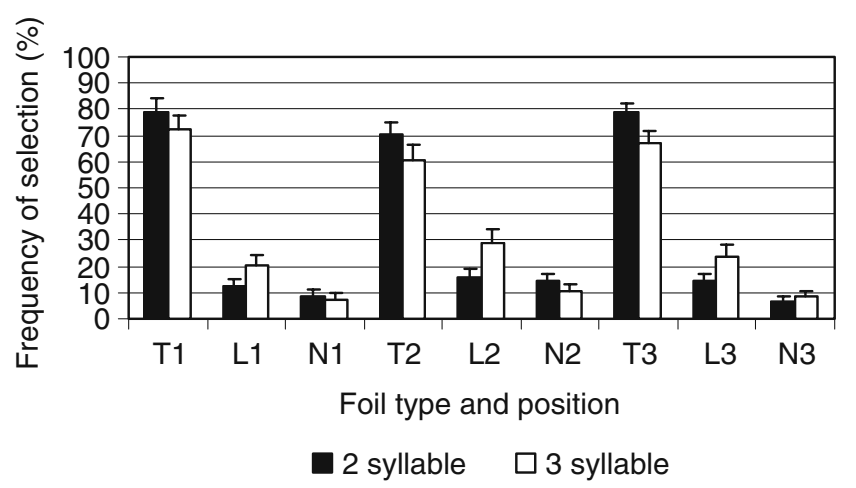

Fig. 2 Percentage selections of target and foils at each position in spoken nonword recognition, for two-syllable and three-syllable stimuli. For labels on the $x$-axis, letters indicate the foil type $(\mathrm{T}=$ target, $\mathrm{L}=$ lexical foil, $\mathrm{N}=$ nonlexical foil) and numbers indicate the position of the foil $(1,2$, or 3). Error bars indicate standard errors 
term lexical and sublexical knowledge, as well as the interaction of the two in the encoding/storage of nonwords. First, the significant decline in target selections for threesyllable nonwords relative to two-syllable nonwords shows the involvement of phonological working memory: Threesyllable nonwords are longer in duration and therefore have to be maintained in phonological working memory for a longer period of time until the target is heard. Children are therefore susceptible to length effects in the spoken recognition of nonwords, whereas adults are not (Frisch et al., 2000). Second, the number of lexical foil selections significantly outweighed the number of nonword foil selections, showing that lexical and sublexical knowledge must be involved in the encoding/storage of nonwords. Third, as nonword length increased, there was a significant increase in lexical foil selections - indicating an interaction between phonological working memory and long-term lexical and sublexical knowledge. Because three-syllable stimuli are more likely to be degraded in quality, there is more emphasis placed on long-term lexical and sublexical knowledge to support their representation in phonological working memory. That is, a decrease in the quality of a stored nonword leads to an increase in the likelihood that a nonword containing a lexical syllable will be erroneously selected as a nonword target.

\section{Nonword repetition}

Verification of data The nonword responses were transcribed into the phonetic alphabet of the CMU Pronouncing Dictionary by the second author. The reliability of the transcriptions was assessed using a pseudorandom sample (to include at least 1 child from each school) of 5 children $(16 \%)$. These samples were subsequently transcribed by (1) a researcher working on an unrelated project who has experience in transcribing nonword repetitions, and (2) a trained linguist. Phoneme-by-phoneme interrater reliability was $87 \%$ for the transcriptions produced by Interrater 1 and $91 \%$ for the transcriptions produced by Interrater 2 .

Repetition analyses We defined a lexical error as any error that involved a syllable in a nonword being changed to a syllable that occurred in the CPWD - that is, we only considered as a lexical error those words that are likely to be in the child's vocabulary. To be as consistent as possible with the spoken nonword recognition results, the percentages of lexical errors were calculated by dividing the number of nonwords repeated with a lexical error by the total number of nonword repetitions in each condition (i.e., number of lexical errors / number of correct nonword repetitions + number of repetitions involving a lexical error + number of repetitions involving a nonlexical error). All analyses in this section used a 2 (nonword length: two or three syllables) $\times 3$ (pause duration: short, medium, or long) repeated measures ANOVA.

Figure 3 shows the percentage of repetitions containing a lexical error for nonwords at each length and pause duration. There was no effect of nonword length $[F(1,30)=0.27, p=$ $\left..609, \eta_{\mathrm{p}}{ }^{2}=.01\right]$ but a significant effect of pause duration $\left[F(2,60)=5.11, p=.009, \eta_{\mathrm{p}}^{2}=.15\right]$, with post-hoc Bonferroni tests indicating that more lexical errors were made at the longest pause duration than at either the short duration $(p=.041)$ or the medium duration $(p=.040)$. These results indicate an interaction between phonological working memory and long-term lexical and sublexical knowledge (e.g., via redintegration). As the representations of nonwords in phonological working memory decrease in quality, the involvement of long-term lexical and sublexical knowledge increases. For the present study, it is at the longest temporal duration that nonwords become sufficiently degraded in quality for long-term lexical and sublexical knowledge to be used as an aid to repetition. However, the same is not true when comparing short and long nonwords, where the proportions of lexical error do not differ. We will return to the issues surrounding the lexical error/nonword length data in the General Discussion.

There was a significant interaction between nonword length and pause duration $\left[F(2,60)=4.18, p=.020, \eta_{\mathrm{p}}{ }^{2}=\right.$ .12] caused by the three-syllable nonwords at a medium pause duration. Because the repetition accuracy at this length and duration was relatively high (see Table 1 later), there was a relatively low number of lexical errors.

We also examined repetition accuracy. A nonword was repeated accurately if all constituent phonemes were correctly articulated, and was regarded as inaccurate if one or more phonemes were incorrectly repeated. Table 1 shows the mean percentage of nonwords correctly repeated for each pause duration and length of nonword. There was a significant effect of nonword length $[F(1,30)=$ 22.72, $\left.p<.001, \eta_{\mathrm{p}}{ }^{2}=.43\right]$, such that two-syllable

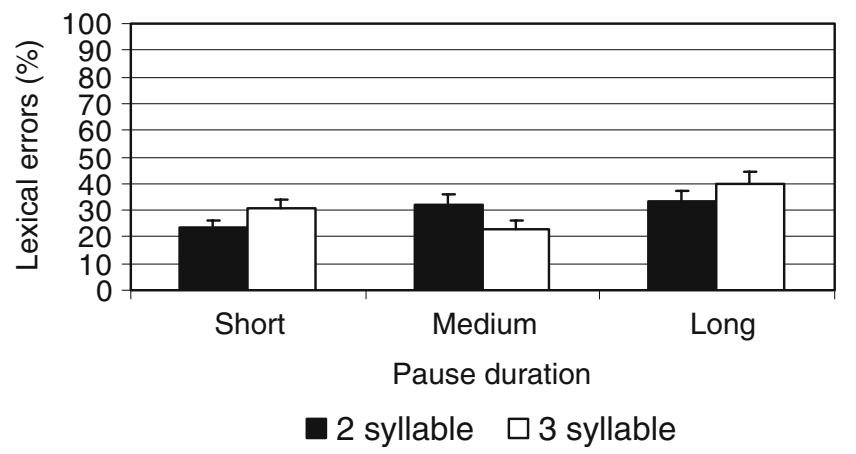

Fig. 3 Percentages of lexical errors at each pause duration in nonword repetition, for two-syllable and three-syllable stimuli. Error bars indicate standard errors 
Table 1 Mean percentage of nonwords repeated accurately for each pause duration and length of nonword (standard deviations in parentheses)

\begin{tabular}{lll}
\hline Pause Duration & \multicolumn{2}{l}{ Nonword Length } \\
\cline { 2 - 3 } & Two Syllables & Three Syllables \\
\hline Short & $48.92(22.33)$ & $30.11(21.70)$ \\
Medium & $40.00(19.32)$ & $44.52(24.06)$ \\
Long & $40.65(18.96)$ & $20.65(20.32)$ \\
\hline
\end{tabular}

nonwords were repeated more accurately than threesyllable nonwords. There was also a significant effect of pause duration $\left[F(2,60)=6.48, p=.003, \eta_{\mathrm{p}}{ }^{2}=.18\right]$, with post-hoc Bonferroni tests indicating greater accuracy for both the short and medium pause durations over the long pause duration ( $p=.036$ and .006 , respectively). However, there was also a significant interaction between nonword length and pause duration $\left[F(2,60)=10.53, p<.001, \eta_{\mathrm{p}}{ }^{2}=\right.$ .26 , caused by the relatively high repetition accuracy for three-syllable nonwords with a medium pause duration.

The accuracy results are similar to the spoken nonword recognition results in that accuracy became lower as nonword length increased. However, in spoken nonword recognition there was no effect of the position of the target foil, whereas in repetition, a long delay until repeating a nonword caused a reduction in accuracy. One possible reason for the lack of a position effect in spoken nonword recognition may be rehearsal. Although the children themselves were unlikely to be rehearsing material (Gathercole \& Adams, 1994), the spoken nonword recognition task provides some rehearsal of information, since the two foils only differ from the target by one phoneme.

Spoken nonword recognition versus repetition

One of the main purposes of the present study was to examine performance differences across spoken nonword recognition and NWR. Below, we first examine lexical errors across spoken nonword recognition and NWR, and second, we examine accuracy. To make the analyses more tractable, the data are collapsed by position/pause duration. We only consider effects involving the type of task, since task comparison is the main area of interest (for errors, all other effects will have been covered earlier).

Figure 4 shows the two- and three-syllable lexical error data for spoken nonword recognition and NWR. A 2 (task type: spoken nonword recognition or NWR) $\times 2$ (nonword length: two or three syllables) mixed ANOVA was carried out on the data, with task type as the between-subjects variable. Importantly, there is a significant effect of task type $\left[F(1,60)=14.17, p<.001, \eta_{\mathrm{p}}{ }^{2}=.19\right]$, showing that spoken nonword recognition involves fewer lexical errors than NWR. Previous research, together with the present study, has shown that lexical errors are prevalent in NWR. However, previous research did not indicate whether the source of lexical errors in NWR should be attributed to the encoding/storage process or the articulation process. We can now address this issue. The design of the present study aimed to minimise any differences in encoding/storage across spoken nonword recognition and NWR. Given that the encoding/storage of material should be similar across the two tasks, the additional lexical error seen in NWR must be produced by lexical and sublexical knowledge infiltrating the articulation process. The encoding/storage and articulation of nonwords are both influenced by lexical and sublexical knowledge.

There is an interaction between task type and nonword length $\left[F(1,60)=5.19, p=.026, \eta_{\mathrm{p}}{ }^{2}=.08\right]$. As Fig. 4 and the individual analyses for each type of task show, for spoken nonword recognition there is an increase in lexical errors as nonword length increases from two to three syllables; the same is not true for NWR, where lexical errors are stable across two and three syllables.

Figure 4 shows the accuracy data for spoken nonword recognition and NWR. There is a clear effect of task type $\left[F(1,60)=63.23, p<.001, \eta_{\mathrm{p}}^{2}=.51\right]$ showing superior accuracy for spoken nonword recognition relative to NWR. There is no interaction between task type and nonword length $\left[F(1,60)=0.35, p=.556, \eta_{\mathrm{p}}^{2}=.01\right]$. Adding an articulatory component causes a significant decrease in accuracy, with accuracy almost halving when moving from spoken nonword recognition to NWR.

\section{Summary}

Previous research has shown long-term lexical and sublexical involvement in NWR performance (e.g., Munson,

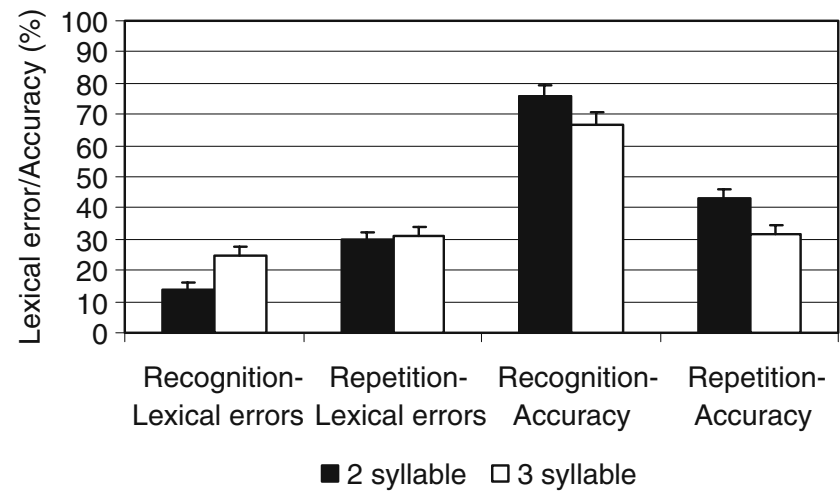

Fig. 4 Lexical errors and accuracy for spoken nonword recognition and repetition, for two-syllable and three-syllable stimuli. Error bars indicate standard errors 
2001; Munson et al., 2005a,b). However, NWR involves various processes, such as encoding/storage and articulation. To our knowledge, no previous study has examined which of the processes involved in NWR are affected by long-term lexical and sublexical knowledge. The present study targeted encoding/storage and articulation within the same nonword study using the same set of stimuli. A spoken nonword recognition task was hypothesised to involve at least a significant encoding/storage component but no articulation component, and a NWR task was hypothesised to involve both encoding/storage and articulation as significant processes. For spoken nonword recognition, there were significantly more lexical errors than nonlexical errors, indicating the influence of long-term lexical and sublexical knowledge in the encoding/storage of nonwords. In NWR, approximately $30 \%$ of repetitions involved a lexical error, indicating involvement of lexical and sublexical knowledge in encoding/storage, articulation, or both. Importantly, there was a significantly higher number of lexical errors for NWR than for spoken nonword recognition. Since the principal difference between the two tasks was an additional process of articulation for NWR, encoding/storage and articulation must both be influenced by long-term lexical and sublexical knowledge.

At the end of the introduction, we outlined three research questions that, to our knowledge, have not yet been addressed in nonword research. The present study has been able to address each of them. First, encoding/storage and articulation are both influenced by lexical and sublexical knowledge. In fact, our results show that almost twice as many lexical errors are produced in NWR than in spoken nonword recognition, illustrating that the addition of an articulation component almost doubles the number of lexical errors.

Second, the influence of lexical and sublexical knowledge increases as the quality of a stored nonword decreases. As the burden of holding a nonword in phonological working memory increases, there is an increased reliance on long-term lexical and sublexical knowledge to bolster the representation of the nonword. In our results for spoken nonword recognition, the selection of lexical foils increased as the length of the nonword increased; for repetition, there were more lexical errors as the delay until repeating a nonword increased. Note that these results occurred even though the nonword stimuli were low in frequency, nonlexical, and nonmorphological-thus showing that long-term lexical and sublexical knowledge is pervasive in tests involving nonwords.

Third, errors in NWR are caused by both encoding/ storage and articulation. Not surprisingly, accuracy was greater when children were asked to recognise spoken nonwords than when they were asked to repeat them. In fact, children were approximately twice as accurate in recognising spoken nonwords as they were in repeating them. However, children still made errors in spoken nonword recognition - approximately half as many as in NWR. Up to half of the errors produced in NWR tasks may therefore be caused by problems in encoding/storing sound patterns rather than in articulating them. Although this assertion must be treated cautiously - the tasks presented here used a delay, and the spoken nonword recognition task was not a pure encoding/storage task, given there was a selection process involved - the findings nevertheless suggest that there is some utility in breaking down the NWR task into its component processes to establish where errors occur.

Our study also tested the redintegration account of NWR. Redintegration predicts that long-term lexical and sublexical knowledge is used at the point of retrieval to aid the representation of decayed nonwords in phonological working memory. As the pause duration until repeating a nonword increases, the number of repetitions involving a lexical error should increase due to greater decay. Similarly, long nonwords would be expected to decay more than short nonwords, due to their length, and hence repetitions of long nonwords should involve a greater number of lexical errors. The results fit with the first of these predictions. As pause duration increased, the number of lexical errors also increased-particularly for the longest pause duration. However, there was no difference between two-syllable and three-syllable nonwords in terms of the number of lexical errors. Our spoken nonword recognition results also present problems for a redintegration account. Since redintegration occurs at the point of retrieval, there should be little influence of lexical and sublexical knowledge for a task that requires minimal retrieval. However, there was a clear effect of lexical and sublexical knowledge during the spoken recognition of nonwords. Redintegration or a similar process therefore must occur earlier than at the point of retrieval. Other researchers have indicated that redintegration may be involved at earlier stages of the repetition process (see Hulme et al., 1997; Roodenrys, Hulme, Lethbridge, Hinton, \& Nimmo, 2002; Thorn et al., 2005).

Finally, there was one interesting finding regarding lexical errors: Length effects were seen for spoken nonword recognition but not for NWR. It would therefore appear that for nonword tasks that primarily involve encoding/storage, the extent of lexical error is determined by the level of decay of a nonword in phonological working memory. However, for tasks that involve an articulatory component, articulation processes override any length effects caused at other stages. There is certainly a precedent for the latter view. Jones et al. (in press) examined NWR in both typically developing children and children with specific language impairment, finding that both groups showed no 
influence of nonword length on lexical error production. In fact, the predictor of lexical errors during repetition was the lexical neighbourhood size of the nonwords involved. In the present study, the nonwords in each condition were matched for neighbourhood size, which is a possible reason that the numbers of lexical errors were equal for both twosyllable and three-syllable nonwords. In nonword repetition, therefore, the articulation process is the key determiner of lexical errors - a process that is primarily influenced by lexical neighbourhood.

The results presented here show that long-term lexical and sublexical knowledge is pervasive in both the spoken recognition and repetition of nonwords, even for those containing low-frequency phoneme sequences. That is, encoding/storage and articulation processes are influenced by long-term lexical and sublexical knowledge, irrespective of the frequency of the nonword biphones. Any theory of NWR performance must explain the interaction process between long-term lexical and sublexical knowledge and phonological working memory - for all types of nonwords and throughout the whole repetition process, including encoding, storage, and articulation.

Acknowledgement The authors thank the Experimental Psychology Society, who funded this research in the form of an Undergraduate Student Research Bursary awarded to the second author. We thank Marco Tamburelli and Sarah Watson for their helpful discussions and their transcriptions of the nonwords for interrater reliability, and Markus Damian and three anonymous reviewers for their useful comments on previous drafts of this article. We also thank the schools and children who participated in this study. Send correspondence about this article to G. Jones, Division of Psychology, Nottingham Trent University, Chaucer Building, Nottingham NG1 5LT, UK (e-mail: gary.jones@ntu.ac.uk).

\section{References}

Archibald, L. M. D., \& Gathercole, S. E. (2006). Nonword repetition: A comparison of tests. Journal of Speech, Language, and Hearing Research, 49, 970-983.

Baddeley, A. D., \& Hitch, G. J. (1974). Working memory. In G. Bower (Ed.), The psychology of learning and motivation: Advances in research and theory (pp. 47-90). New York: Academic Press.

Baddeley, A. D., Papagno, C., \& Vallar, G. (1988). When long term learning depends on short-term storage. Journal of Memory and Language, 27, 586-595.

Balota, D. A., \& Chumbley, J. I. (1985). The locus of word-frequency effects in the pronunciation task: Lexical access and/or production? Journal of Memory and Language, 24, 89-106.

Brysbaert, M., Lange, M., \& Wijnendaele, I. V. (2000). The effects of age-of-acquisition and frequency-of-occurrence in visual word recognition: Further evidence from the Dutch language. European Journal of Cognitive Psychology, 12, 65-85.

Cheung, H. (1996). Nonword span as a unique predictor of secondlanguage vocabulary learning. Developmental Psychology, 32, 867-873.
Coady, J. A., \& Aslin, R. N. (2004). Young children's sensitivity to probabilistic phonotactics in the developing lexicon. Journal of Experimental Child Psychology, 89, 183-213.

Dollaghan, C. A., Biber, M. E., \& Campbell, T. F. (1995). Lexical influences on nonword repetition. Applied Psycholinguistics, 16, 211-222.

Dunn, L. M., Dunn, L. M., Whetton, C., \& Burley, J. (1997). The British picture vocabulary scale ( 2 nd ed.). Windsor: NFERNelson.

Forster, K. I., \& Chambers, S. M. (1973). Lexical access and naming time. Journal of Verbal Learning Behavior, 12, 627-635.

Frederikson, J. R., \& Kroll, J. F. (1976). Spelling and sound: Approaches to the internal lexicon. Journal of Experimental Psychology: Human Perception and Performance, 2, 361-379.

Frisch, S. A., Large, N. R., \& Pisoni, D. B. (2000). Perception of wordlikeness: Effects of segment probability and length on the processing of nonwords. Journal of Memory and Language, 42, 481-496.

Gathercole, S. E. (1995). Is nonword repetition a test of phonological memory or long-term knowledge? It all depends on the nonwords. Memory \& Cognition, 23, 83-94.

Gathercole, S. E. (2006). Nonword repetition and word learning: The nature of the relationship. Applied Psycholinguistics, 27, 513543.

Gathercole, S. E., \& Adams, A.-M. (1993). Phonological working memory in very young children. Developmental Psychology, 29, $770-778$.

Gathercole, S. E., \& Adams, A.-M. (1994). Children's phonological working memory: Contributions of long-term knowledge and rehearsal. Journal of Memory and Language, 33, 672-688.

Gathercole, S. E., \& Baddeley, A. D. (1989). Evaluation of the role of phonological STM in the development of vocabulary in children: A longitudinal study. Journal of Memory and Language, 28, 200-213.

Gathercole, S. E., Frankish, C. R., Pickering, S. J., \& Peaker, S. (1999). Phonotactic influences on short-term memory. Journal of Experimental Psychology. Learning, Memory, and Cognition, 25, 84-95.

Gathercole, S. E., \& Pickering, S. J. (1999). Estimating the capacity of phonological short-term memory. International Journal of Psychology, 34, 378-382.

Gathercole, S. E., Willis, C. S., Emslie, H., \& Baddeley, A. D. (1992). Phonological memory and vocabulary development during the early school years: A longitudinal study. Developmental Psychology, 28, 887-898.

Gupta, P. (2003). Examining the relationship between word learning, nonword repetition, and immediate serial recall in adults. The Quarterly Journal of Experimental Psychology, 56A, 1213-1236.

Gupta, P. (2005). Primacy and recency in nonword repetition. Memory, 13, 318-324.

Hulme, C., Maughan, S., \& Brown, G. D. A. (1991). Memory for familiar and unfamiliar words: Evidence for a long-term memory contribution to short-term memory span. Journal of Memory and Language, 30, 685-701.

Hulme, C., Roodenrys, S., Schweickert, R., Brown, G. D. A., Martin, S., \& Stuart, G. (1997). Word-frequency effects on short-term memory tasks: Evidence for a redintegration process in immediate serial recall. Journal of Experimental Psychology. Learning, Memory, and Cognition, 23, 1217-1232.

Jones, G., Gobet, F., \& Pine, J. M. (2007). Linking working memory and long-term memory: A computational model of the learning of new words. Developmental Science, 10, 853-873.

Jones, G., Tamburelli, M., Watson, S. E., Gobet, F., \& Pine, J. M. (in press). Lexicality and frequency in specific language impairment: Accuracy and error data from two nonword repetition tasks. Journal of Speech, Language, and Hearing Research. 
Jusczyk, P. W., Luce, P. A., \& Charles-Luce, J. (1994). Infants' sensitivity to phonotactic patterns in the native language. Journal of Memory and Language, 33, 630-645.

Masterson, J., Stuart, M., Dixon, M., \& Lovejoy, S. (2010). Children's printed word database: Continuities and changes over time in children's early reading vocabulary. British Journal of Psychology, 101, 221-242.

Munson, B. (2001). Phonological pattern frequency and speech production in adults and children. Journal of Speech, Language, and Hearing Research, 44, 778-792.

Munson, B., Edwards, J., \& Beckman, M. E. (2005). Relationships between nonword repetition accuracy and other measures of linguistic development in children with phonological disorders. Journal of Speech, Language, and Hearing Research, 48, 61-78.

Munson, B., Kurtz, B. A., \& Windsor, J. (2005). The influence of vocabulary size, phonotactic probability, and wordlikeness on nonword repetitions of children with and without specific language impairment. Journal of Speech, Language, and Hearing Research, 48, 1033-1047.

Norris, D., McQueen, J. M., \& Cutler, A. (2003). Perceptual learning in speech. Cognitive Psychology, 47, 204-238.

Pitt, M. A., \& McQueen, J. M. (1998). Is compensation for coarticulation mediated by the lexicon? Journal of Memory and Language, 39, 347-370.
Roodenrys, S., Hulme, C., Lethbridge, A., Hinton, M., \& Nimmo, L. M. (2002). Word-frequency and phonological-neighborhood effects on verbal short-term memory. Journal of Experimental Psychology. Learning, Memory, and Cognition, 28, 1019-1034.

Rossmeissl, P. G., \& Theios, J. (1982). Identification and pronunciation effects in a verbal reaction time task for words, pseudowords, and letters. Memory \& Cognition, 10, 443-450.

Schweickert, R. (1993). A multinomial processing tree model for degradation and redintegration in immediate recall. Memory \& Cognition, 21, 168-175.

Swingley, D., \& Aslin, R. N. (2007). Lexical competition in young children's word learning. Cognitive Psychology, 54, 99-132.

Thorn, A. S. C., Gathercole, S. E., \& Frankish, C. R. (2005). Redintegration and the benefits of long-term knowledge in verbal short-term memory: An evaluation of Schweickert's (1993) multinomial processing tree model. Cognitive Psychology, 50, $133-158$.

Vitevitch, M. S., \& Luce, P. A. (1998). When words compete: Levels of processing in perception of spoken words. Psychological Science, 9, 325-329.

Vitevitch, M. S., Luce, P. A., Charles-Luce, J., \& Kemmerer, D. (1997). Phonotactics and syllable stress: Implications for the processing of spoken nonsense words. Language and Speech, 40, $47-62$. 